Article

\title{
A Flexible Maximum Power Point Tracking Control Strategy Considering Both Conversion Efficiency and Power Fluctuation for Large-inertia Wind Turbines
}

\author{
Hongmin Meng * (D), Tingting Yang, Ji-zhen Liu and Zhongwei Lin \\ State Key Laboratory of Alternate Electrical Power System with Renewable Energy Sources, \\ North China Electric Power University, Beijing 102206, China; yangtingting@ncepu.edu.cn (T.Y.); \\ ljz@ncepu.edu.cn (J.-z.L.); linzhongwei2003@tom.com (Z.L.) \\ * Correspondence: lymhm310@gmail.com; Tel.: +86-010-61772965 \\ Academic Editor: Frede Blaabjerg \\ Received: 13 June 2017; Accepted: 4 July 2017; Published: 6 July 2017
}

\begin{abstract}
In wind turbine control, maximum power point tracking (MPPT) control is the main control mode for partial-load regimes. Efficiency potentiation of energy conversion and power smoothing are both two important control objectives in partial-load regime. However, on the one hand, low power fluctuation signifies inefficiency of energy conversion. On the other hand, enhancing efficiency may increase output power fluctuation as well. Thus the two objectives are contradictory and difficult to balance. This paper proposes a flexible MPPT control framework to improve the performance of both conversion efficiency and power smoothing, by adaptively compensating the torque reference value. The compensation was determined by a proposed model predictive control (MPC) method with dynamic weights in the cost function, which improved control performance. The computational burden of the MPC solver was reduced by transforming the cost function representation. Theoretical analysis proved the good stability and robustness. Simulation results showed that the proposed method not only kept efficiency at a high level, but also reduced power fluctuations as much as possible. Therefore, the proposed method could improve wind farm profits and power grid reliability.
\end{abstract}

Keywords: wind turbine; maximum power point tracking; MPPT; conversion efficiency; power smoothing; power fluctuation; multi-objective control; cost function

\section{Introduction}

With more and more attention being paid to environmental problems, wind energy has become one of the most important renewable energy sources to develop and utilize [1,2]. Although wind turbine control techniques have been developed for several decades, there are still many tough control problems to be solved. Generally, wind turbine control is divided into two parts: partial-load regime (below rated wind speed) and full-load regime (above rated wind speed). In full-load regime, power smoothing is the main control objective, and it is unnecessary to consider energy conversion efficiency. In partial-load regime, maximum power point tracking (MPPT) is the main operation mode [3,4]. The control objectives in partial-load regime consist of achieving high wind energy conversion efficiency, smoothing output power, diminishing mechanical loads, riding through fault conditions, etc. In these objectives, efficiency potentiation and power smoothing have been studied in-depth separately $[2,5,6]$. However, on one hand, with the increase of turbine inertia, enhancing conversion efficiency leads to higher fluctuations of generator power. On the other hand, power smoothing without energy storage devices may cause efficiency loss. Although many power smoothing methods based on storage devices are able to reduce this contradiction, the cost of energy storage devices is expensive. Therefore, it is 
essential to balance conversion efficiency and power smoothing in partial-load regime without energy storage devices.

Balancing conversion efficiency and power smoothing is multi-objective optimization (MOO) problem. A novel MPPT framework employing fuzzy inference system (FIS) was proposed in [3] to consider these multiple objectives. The captured energy efficiency is enhanced, and power fluctuation is suppressed as much as possible, but FIS requires a priori artificial experience, and it is complex to establish fuzzy memberships. Other advanced control algorithms, such as adaptive control [7], robust control [8] and nonlinear control [9], are also employed in wind energy conversion systems (WECSs). Reference [7] proposed an adaptive MPPT control based on a network-based reinforcement learning method to deal with the changes of operating environment. A robust nonlinear control is presented in [8] to maximize energy conversion which allows maximization without exacting wind turbine model knowledge. In [9], the dynamics aspects of the wind and aeroturbine are taken into consideration by proposing nonlinear static and dynamic state feedback controllers. Although the aforementioned advanced control methods are effective for MPPT control, they cannot handle the problems with linear or nonlinear constraints. Model predictive control (MPC) is widely adopted in engineering for multi-objective problems with linear or nonlinear constraints. It has been employed in WECS for generator control [10,11], power converter control [12,13], wind-battery hybrid control [14] and other multi-objective control [15]. Mechanical load and conversion efficiency are considered in $[16,17]$. In [18,19], MPC is used to maximize energy conversion, mitigate drive train loads in both partial-load and full-load regime. However, power smoothing is only considered in full-load regime. In fact, it is essential to suppress extra fluctuations of generator power in partial-load regime. The stability of MPC with receding horizon is guaranteed by employing a terminal quality constraint [20]. The modeling of local linearization state-space equations is important for MPC design. Mostafa [18] presented a MPC method where the linear models are switched according to wind speed condition. A fuzzy model- based multivariable predictive control approach is also proposed in [21] to improve control performance. In the state-space models for the studies above, the wind speed within the control horizon is often regarded as constant, and wind variations are omitted in the optimization solving process. However, power fluctuations are closely related to the wind variations in current control horizon, and reducing fluctuations requires taking wind speed variations into account. The weights in the MPC value function play a key role in the trade-off between the multiple objectives. Flexible weight tuning strategies can help improve control performances. A tuning approach based on the computation of sensitivity tables is proposed for tradeoff in [22]. In [23], weights are adjusted in response to the variable wind conditions and operational requirements by classifying different wind speed scenarios.

In this paper, a balancing strategy for large-inertia WECSs is proposed based on a flexible MPPT control framework employing a model predictive method. Energy conversion efficiency and power smoothing are both considered under partial-load regime. Firstly, the relationship between conversion efficiency, generator power smoothing and turbine inertia is analyzed. Conversion efficiency potentiation may increase the generator power fluctuation. For a large inertia turbine, increasing the same conversion efficiency brings much larger fluctuation than for a small inertia turbine. Thus, it is necessary to balance and alleviate extra power fluctuation at no or very low conversion efficiency cost. Secondly, a new control framework based on classical optimal torque control (OTC) is presented to compensate the torque reference in order to regulate the dynamic performance of the system by adjusting the compensation gain. For large-inertia wind turbines, different compensation gains may lead to different conversion efficiencies, as well as power fluctuations. Then, thirdly, to determine the compensation gain, the MPC method is proposed to solve the optimization problem considering both conversion efficiency and power smoothing. The computational burden to solve the optimization problem is reduced by transforming the cost function representation. Besides, dynamic weights in the cost function are presented to improve the control performance. Benefiting from the proposed control framework, the stability of the control system is guaranteed without any 
terminal constraints, which are usually adopted in traditional open-loop MPC control methods. In addition, the proposed control has good robust performance, and the stability is kept despite uncertain parameters and unmodeled dynamics in the MPC. The proposed method not only maintains the energy conversion efficiency at a high level, but also reduces power fluctuations as much as possible. Therefore, it can increase the economic profits of wind farm operators and improve the reliability of power systems.

The remainder of this paper is organized as follows: Section 2 presents brief model descriptions used for our state-space model and simulations. In Section 3, the relationship between power fluctuations, conversion efficiency and turbine inertia is analyzed. Details of the proposed method are discussed in Section 4, including the proposed control framework, MPC implementation, dynamic weights and stability and robustness analysis. Finally, simulation studies are carried out to verify its effectiveness in Section 5. Section 6 presents the conclusions that summarize the main contributions of this paper.

\section{Brief Model Description}

\subsection{Overall WECS Model}

Figure 1 outlines a simplified WECS. The main components of a WECS consist of wind turbine system (wind turbine model), transmission system (drive train model) and electrical system (generator model). Firstly, wind turbine converts wind power into captured mechanical power. Secondly, the transmission system transmits the mechanical power captured by the rotor to the generator in the electrical system. A gearbox is included in the transmission system to increase the rotor speed to values more suitable for driving the generator. Note that gear box will be removed in a direct-driven wind turbine. Thirdly, the generator transforms mechanical power into electrical power. Its electrical terminals are connected to the power system. Note that power electronics devices, which are usually used to control generator output power, are not illustrated in Figure 1.

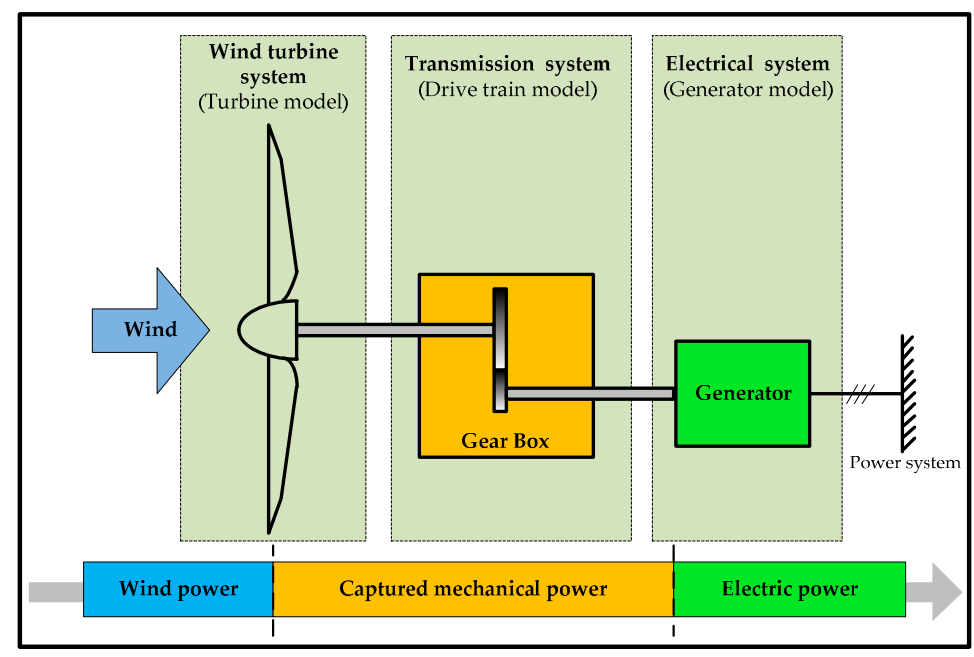

Figure 1. Overall schematic of a wind energy conversion system.

\subsection{Effective Wind Speed Model}

Since effective wind speed contains the rotational sampling effect of the wind turbine, it can be used for a thorough assessment of the designed controller performance. The effective wind speed can be modeled as a sum of two components [18,24]:

$$
V_{w}=V_{\text {mean }}+V_{\text {turb }}
$$


where $V_{\text {mean }}$ is an average wind speed component that varies slowly in the long-term time domain. $V_{\text {turb }}$ is a turbulent component that changes rapidly. The turbulent component is obtained by passing white noise through three cascaded filters [25], $H_{\mathrm{F}}(w), H_{\mathrm{SF}}(w)$ and $H_{\mathrm{RSF}}(w)$. The first filter $H_{\mathrm{F}}(w)$ is used to obtain the turbulence by approximating the von Karman spectrum or the Kaimal spectrum. The filters $H_{\mathrm{SF}}(w)$ and $H_{\mathrm{RSF}}(w)$ are included to take account of the rotational sampling effect on the turbulence derived from filter $H_{\mathrm{F}}(w)$. The filter $H_{\mathrm{SF}}(w)$, called spatial filter, attenuates the high-frequency components of the turbulence. The filter $H_{\mathrm{RSF}}(w)$ models the effect of rotational sampling, and amplifies those components with frequencies close to triple the turbine speed (3P frequency). Details of effective wind speed calculation can be found in [25].

\subsection{Wind Turbine Model}

Wind energy is captured by the wind turbine, and the captured mechanical power $P_{w t}$ can be written as:

$$
P_{w t}=0.5 \rho \pi R_{w}{ }^{2} V_{w}^{3} C_{p}(\lambda, \beta)
$$

where $\rho$ is the air density, $R_{w}$ is the radius of the turbine rotor and $V_{w}$ is the wind speed. $C_{p}$ is a power coefficient that denotes the wind energy conversion efficiency. It is the function of tip-speed ratio $\lambda=\omega_{r} R_{w} / V_{w}$ and pitch angle $\beta . \omega_{r}$ is the rotor speed.

\subsection{Drive Train Model}

The drive train model illustrated in Figure 1 can be equivalent to a single-mass shaft model coupling two parts: turbine side and generator side, as shown in Figure 2. Inertia $J$ is the equivalent inertia of single mass that including both turbine rotor and generator rotor inertia. $N_{\text {gear }}$ is the gear ratio, $\omega_{r}$ is rotor speed, $T_{m}$ and $T_{e}$ are the torques on each side of the transmission system. The equivalent values of inertia and generator torque need to take gear ratio into account.

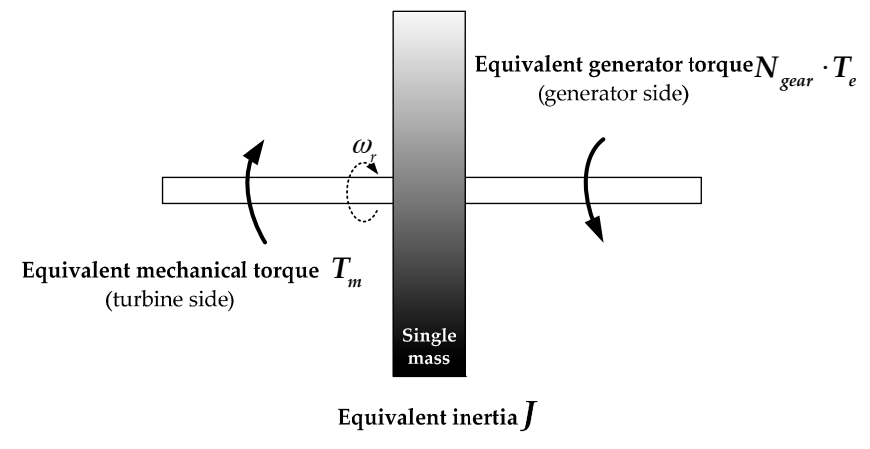

Figure 2. One-mass drive train model.

According to Newton's second law of rotation, the drive train model can be expressed as:

$$
J \dot{\omega}_{r}=T_{m}-N_{\text {gear }} \cdot T_{e}
$$

where $J$ is the equivalence of the entire inertia, $N_{\text {gear }}$ is the gear ratio, $T_{m}$ is the mechanical torque, $T_{e}$ is the generator torque, $\dot{\omega}_{r}$ is the angular acceleration of the turbine rotor. For a direct-drive wind turbine, $N_{\text {gear }}=1$.

\subsection{Generator Model}

To decouple active and reactive power control, a $d-q$ synchronous reference frame is employed to express the voltage and flux equations: 


$$
\begin{gathered}
\left\{\begin{array}{l}
u_{d s}=-r_{s} i_{d s}-\omega_{s} \psi_{q s}+\frac{\mathrm{d} \psi_{d s}}{\mathrm{~d} t} \\
u_{q s}=-r_{s} i_{q s}+\omega_{s} \psi_{d s}+\frac{\mathrm{d} \psi_{q s} \mathrm{~d} t}{\mathrm{~d} t} \\
u_{d r}=r_{r} i_{d r}-s \omega_{s} \psi_{q r}+\frac{\mathrm{d} \psi_{d r}}{\mathrm{~d} t} \\
u_{q r}=r_{r} i_{q r}+s \omega_{s} \psi_{d r}+\frac{\mathrm{d} \psi_{q r}}{\mathrm{~d} t}
\end{array}\right. \\
\left\{\begin{array}{l}
\psi_{d s}=-\left(L_{s \sigma}+L_{m}\right) i_{d s}+L_{m} i_{d r} \\
\psi_{q s}=-\left(L_{s \sigma}+L_{m}\right) i_{q s}+L_{m} i_{q r} \\
\psi_{d r}=-\left(L_{r \sigma}+L_{m}\right) i_{d r}+L_{m} i_{d s} \\
\psi_{q r}=-\left(L_{r \sigma}+L_{m}\right) i_{q r}+L_{m} i_{q s}
\end{array}\right.
\end{gathered}
$$

where $\omega_{s}$ is the synchronous speed, $u$ is the voltage, $i$ is the current, $r$ is the resistance, $\psi$ is the flux, $L_{m}$ is the mutual inductance, $L_{\sigma}$ is the leakage inductance. Subscripts $d, q$ denote the $d$-axis and $q$-axis, respectively. Subscripts $s$ and $r$ denote the generator stator and rotor, respectively.

\section{Analysis of Wind Turbine Characteristic}

Multiplying both sides of Equation (3) by the rotor speed $\omega_{r}$, the relation between captured mechanical power $P_{w t}$ and output electric power $P_{e}$ is expressed as:

$$
P_{e}=P_{w t}-P_{\text {rotor }}
$$

where $P_{\text {rotor }}=J \dot{\omega}_{r} \omega_{r}$. According to Equation (6), the captured $P_{w t}$ is divided into two parts, most of the power is converted into the generator power $P_{e}$, then higher variations of $P_{w t}$ result in the fluctuations of electric power $P_{e}$. The rest of $P_{\text {rotor }}$ is stored as rotational kinetic energy. It can be regulated by controlling the rotor speed $\omega_{r}$ and angular acceleration of the rotor $\dot{\omega}_{r}$ in coordination to reduce the fluctuation of $P_{e}[26,27]$. Thus $P_{\text {rotor }}$ supplies the capability to balance efficiency and power smoothing.

According to Equation(6), enhancing the efficiency may increase the fluctuations of the output power. To study the relationship between turbine inertia, power fluctuations and conversion efficiency, studies are carried out based on the MPPT strategy in [5], which is a flexible way to change conversion efficiency by tuning a single control parameter. The first case is to investigate the variation trend of power fluctuations as the conversion efficiency is getting higher under the same turbine inertia conditions. The results in Figure 3 show that a higher efficiency leads to larger power fluctuations with the same turbine inertia. The second case is carried out to study how the power fluctuations change with larger turbine inertia when the conversion efficiencies are kept constant. The results in Figure 4 indicate that, to achieve the same conversion efficiency, a wind turbine with larger inertia would cause higher power fluctuations.

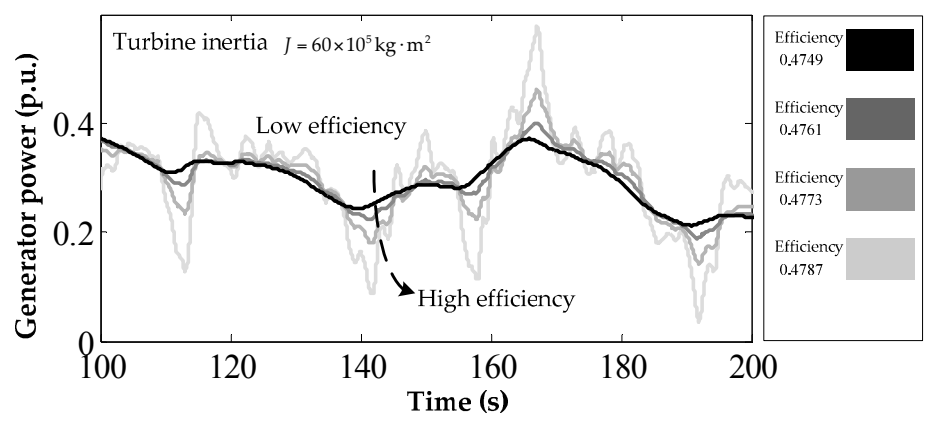

Figure 3. Power fluctuations with different conversion efficiency in the same turbine. 


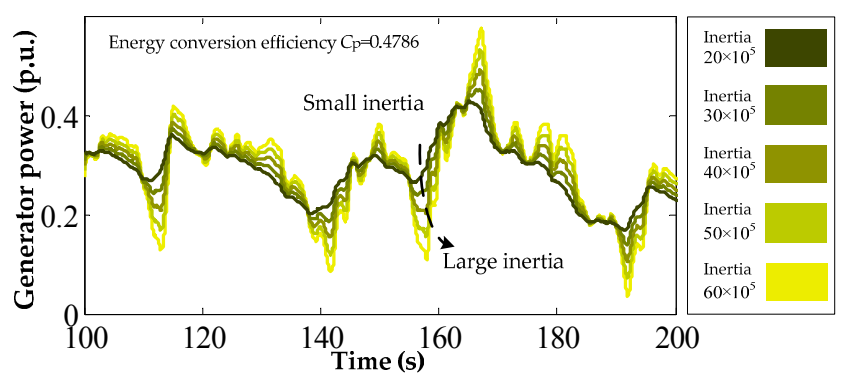

Figure 4. Power fluctuations with different turbine inertia under the same conversion efficiency.

The same MPPT strategy in [5] is used for these analyses. For different studies, only one control parameter is tuned respectively to regulate the conversion efficiency. It is improved based on optimal torque control, which is one of the most widely used MPPT control strategies in wind turbine engineering for its simple implementation and low power fluctuations [28]. A novel proportional control loop is added in this classical optimal torque control scheme to improve the dynamic performance of the system. The proportional gain $K_{p}$ in this control loop determines the wind energy conversion efficiency. By tuning the single control parameter (proportional gain $K_{p}$ ) conversion efficiency is changed flexibly. It is not important to determine which MPPT strategy is adopted for this study above because the variation trends of efficiency, fluctuations and inertia are similar for different control strategies. The reason for employing the MPPT strategy in [5] is that, this strategy is flexible to change conversion efficiency by tuning only single control parameter. Although there may be some kinds of MPPT strategy based on generator speed that could smooth output power utilizing large inertia, the conversion efficiency would decrease significantly. Because rotor speed is controlled to smooth output power, and it cannot track rapid wind variations. This defect would become more evident with larger turbine inertia. Therefore, for the same MPPT strategy, it is hard to maintain both efficiency and fluctuation level unchanged as turbine inertia getting larger. In general, large-inertia wind turbine brings more complex problems in balancing both power smoothing and conversion efficiency enhancement.

\section{Proposed Method}

\subsection{Description of the Proposed Framework}

The MPPT control framework employing a model predictive method is proposed to derive generator torque reference $T_{e}^{*}$ for a closed-loop system. As shown in Figure 5, the left side in the grey area is the proposed method.

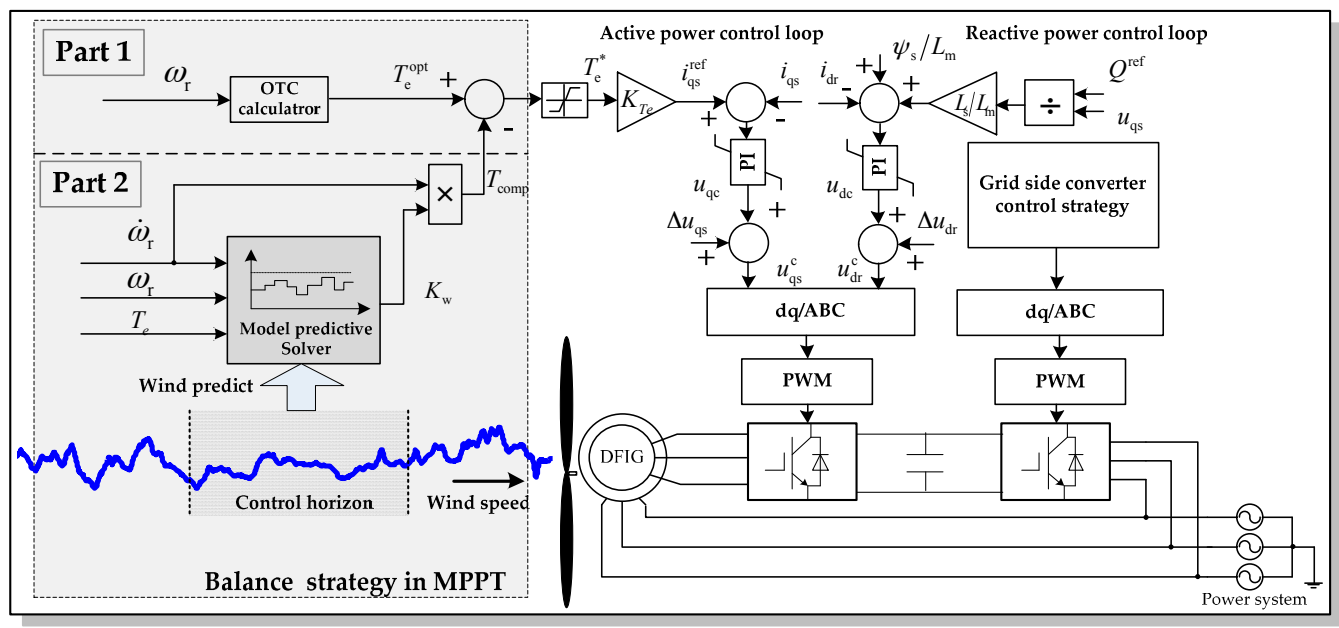

Figure 5. Proposed control framework with predictive method. 
The right side of Figure 5 is the decoupling control in the $d-q$ synchronous reference frame for the generator side converter (GSC), which is employed to control the active power and reactive power independently. The active power is the main component of output power that influences the frequency of the power system. In a normal operating situation, the value of reactive power is usually kept at a low level. There are two main proportional-integral (PI) control loops in the GSC: the active power control loop and the reactive power control loop. The active power is determined by $i_{q s}$ control and the reactive power is determined by $i_{d s}$ control. Details of decoupling control can be found in [3]. It is noted that the generator power discussed in this paper is active power, and the reactive power is set to zero.

The proposed method illustrated in Figure 5 consists of two parts, Part 1 and Part 2. Part 1 is a classical OTC MPPT calculator. OTC is one of the most widely employed MPPT strategies for its good stability, control performance $[3,28]$ and simple implementation. The output of the OTC calculator is expressed as:

$$
T_{e}^{o p t}=K_{o p t} \cdot \omega_{r}^{2} / N_{\text {gear }}
$$

where $K_{o p t}=0.5 \rho \pi R_{w}^{5} C_{p \max } / \lambda_{o p t}^{3} . \lambda_{\text {opt }}$ is the optimum tip-speed ratio. From (7), $T_{e}^{o p t}$ is a function of rotor speed. Since large inertia leads to slow dynamic behavior of the rotor speed $\omega_{r}, T_{e}^{o p t}$ varies slowly without compensation $T_{\text {comp }}$, and the control system does not track wind speed variation. As a result, the conversion efficiency of OTC is reduced significantly, but the capability of power smoothing is improved apparently.

Thus, Part 2 in Figure 5 is designed to take advantage of this and make up the disadvantages discussed above. Compensation torque $T_{\text {comp }}$ is derived depending on rotor acceleration and compensation gain:

$$
T_{\text {comp }}=K_{w} \cdot \dot{\omega}_{r}
$$

Then, the final generator reference is derived after compensation:

$$
T_{e}^{*}=T_{e}^{o p t}-T_{\text {comp }}
$$

Since $\dot{\omega}_{r}$ equals to zero when the wind turbine operates in steady state $\left(C_{p}\right.$ achieves a maximum value), the compensation of Part 2 is only activated during transient processes where the system deviates from its steady-state operation point $(\mathrm{OP}) . K_{w}$ is regulated by a model predictive method considering both conversion efficiency and power smoothing.

Figure 6 is the locus of OPs for classical OTC and proposed control framework, assuming that wind speed steps from $7 \mathrm{~m} / \mathrm{s}$ to $11 \mathrm{~m} / \mathrm{s}$. Figure $6 \mathrm{a}, \mathrm{b}$ is coincident, and the only difference is that rotor acceleration is shown in Figure 6b.
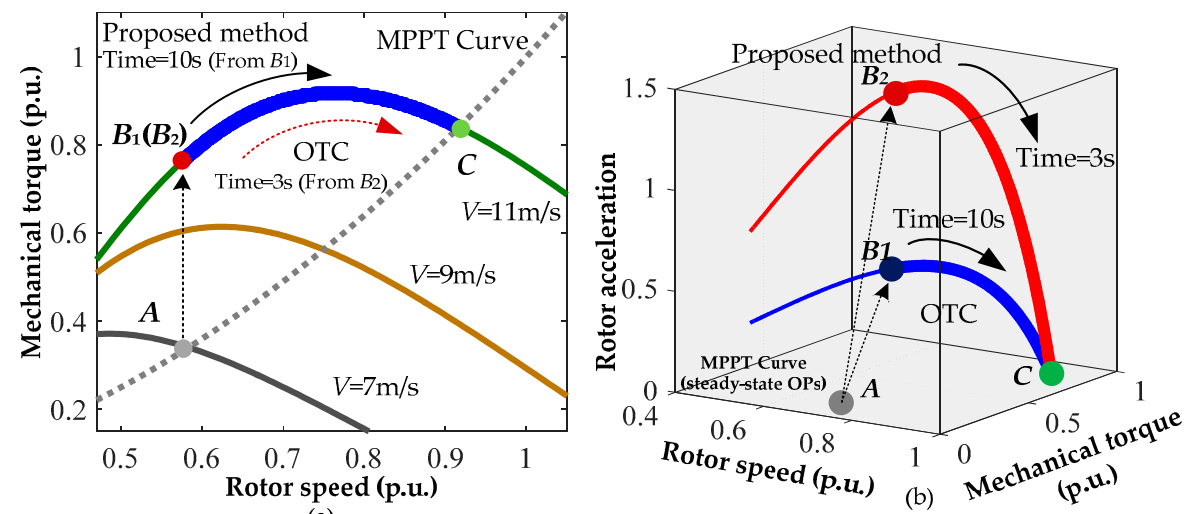

(a)

Figure 6. Moving locus of operation points, (a) 2D view; (b) 3D view with rotor acceleration included. 
The blue curve is the locus of classical OTC, the red curve is the locus of the proposed control framework. When the wind speed changes, the operation points step from $A$ to $B_{1}$, and $B_{2}$, respectively. The rotor acceleration of $B_{2}$ is much larger than that of $B_{1}$. This means that the OP of the proposed method reaches steady-state point $C$ faster (time $=3 \mathrm{~s}$ ) than that of classical OTC method (time $=10 \mathrm{~s}$ ). Therefore, the proposed control framework improves the dynamic performance of control system, and as a result, increases the conversion efficiency of the wind turbine. This effect all depends on $K_{w}$. A larger $K_{w}$ means higher efficiency. However, it also brings higher power fluctuations. Therefore, it is essential to regulate $K_{w}$ reasonably in order to balance the efficiency enhancing and power smoothing capabilities according to the current operating conditions. In this paper, the optimal $K_{w}$ is derived by a model predictive method.

\subsection{Procedures of MPC Implement}

In the proposed control framework discussed above, the model predictive solver plays a key role in determining the value of compensation gain $K_{w}$. MPC is widely used to handle multi-objective control problems in multi-input multi-output (MIMO) systems. The procedure of MPC design is as follows.

- The input, output variables and control signals need to be clear. Linear dynamic state-space model also requires to be established. Simplification of the model is necessary.

- The future output sequence within the control horizon is predicted. The optimized control sequence $\boldsymbol{U}(k)=\left[\boldsymbol{u}(k), \ldots, \boldsymbol{u}\left(k+N_{c}-1\right)\right]^{\mathrm{T}}$ is derived from solving a constrained problem expressed as a value function at each sampling time.

- The first control signal in the sequence is sent to the system. The optimization will be repeated and updated with predictive horizon rolling.

The flow chart of this procedure is presented in Figure 7.

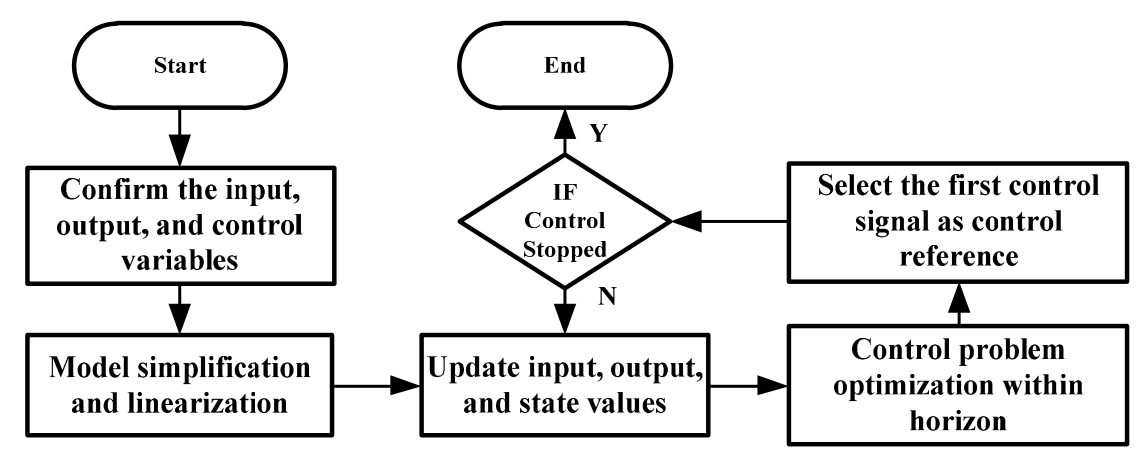

Figure 7. Flow chart of MPC implementation procedure.

In classical MPC, system stability is guaranteed by employing terminal quality constraints. The optimization is realized by solving a quadratic programming $(\mathrm{QP})$ problem on line to derive control sequence, which brings about a great amount of calculation.

\subsection{State-Space Model for Predictive Control}

To reduce the computing burden, simplification of the detailed model is necessary. The generator model can be simplified as a first-order model to represent the electrical dynamics [18]:

$$
\dot{T}_{e}=\frac{1}{\tau_{e}}\left(T_{e}^{*}-T_{e}\right)
$$

where $T_{e}^{*}$ is the generator torque reference, $\tau_{e}$ is the equivalent time constant. According to Equations (3) and (10), the simplified model is nonlinear. Assuming that $x=\bar{x}+\delta x, \bar{x}$ is the steady state under a 
certain wind speed condition, and $\delta x$ is the deviation from $\bar{x}$, linearizing the simplified Equations in (3) and (10) yields:

$$
\left\{\begin{array}{c}
J \delta \dot{\omega}_{r}=\Gamma_{T w} \delta \omega_{r}-N_{g e a r} \cdot \delta T_{e}+\Gamma_{T b} \delta \beta+\Gamma_{T v} \delta V_{w} \\
\tau_{e} \delta \dot{T}_{e}=\delta T_{e}^{*}-\delta T_{e}
\end{array}\right.
$$

where $\Gamma_{T w}=\left.\frac{\partial T_{m}}{\partial \omega_{r}}\right|_{\left(v_{w 0}, \beta_{0}\right)^{\prime}} \Gamma_{T v}=\left.\frac{\partial T_{m}}{\partial V_{w}}\right|_{\left(\omega_{r 0}, \beta_{0}\right)^{\prime}} \Gamma_{T b}=\left.\frac{\partial T_{m}}{\partial \beta}\right|_{\left(\omega_{r 0}, V_{w 0}\right)}$.

Since this research focuses on the MPPT below rated wind speed, then the pitch angle is fixed, $\delta \beta$ is set to zero.

The state-space model for predictive method is given as:

$$
\left\{\begin{array}{c}
\dot{\mathbf{X}}(t)=\left[\begin{array}{cc}
\frac{1}{J} \Gamma_{T w} & -\frac{1}{J} N_{\text {gear }} \\
0 & -\frac{1}{\tau_{e}}
\end{array}\right] \mathbf{X}(t)+\left[\begin{array}{c}
0 \\
\frac{1}{\tau_{e}}
\end{array}\right] \mathbf{u}(t)+\frac{1}{J} \Gamma_{T v} \mathbf{d}(t) \\
\mathbf{Y}(t)=[0,1] \mathbf{X}(t)
\end{array}\right.
$$

where $\mathbf{X}=\left[\boldsymbol{\omega}_{r}, \mathbf{T}_{e}\right], \mathbf{u}=\mathbf{T}_{e}^{*}, \mathbf{d}=\delta \mathbf{V}_{w}$.

Discretizing the continuous model in Equation (12), the state-space equation is expressed as:

$$
\left\{\begin{array}{c}
\mathbf{X}(k+1)=\mathbf{A X}(k)+\mathbf{B u}(k)+\mathbf{E d}(k) \\
\mathbf{Y}(k)=\mathbf{C X}(k)
\end{array}\right.
$$

The cost function for the predictive method is:

$$
J_{\min }=\min [\underbrace{\sum_{i=1}^{N_{c}}\left\|\mathbf{e}_{r}(k+i)\right\|_{\mathbf{M}_{\mathbf{1}}}^{2}}_{\text {Efficiency }}+\underbrace{\sum_{i=1}^{N_{c}}\left\|\Delta \mathbf{e}_{\mathbf{P}}(k+i)\right\|_{\mathbf{M}_{\mathbf{2}}}^{2}}_{\text {Fluctuations }}+\underbrace{\left\|\Delta \mathbf{K}_{w}(k)\right\|_{\mathbf{M}_{\mathbf{3}}}^{2}}_{\text {Increment }}]
$$

subject to $\omega_{r}^{\min } \leq \omega_{r}(k+i) \leq \omega_{r}^{\max }, \dot{\boldsymbol{\omega}}_{r}(k+i) \leq\left|\dot{\omega}_{r}^{\max }\right|, 0 \leq \mathbf{T}_{e}(k+i) \leq T_{e}^{\max }, 0 \leq$ $\mathbf{P}_{\mathbf{e}}(k+i) \leq P_{e}^{\text {rated }}$.

Here, $N_{\mathrm{c}}$ is the control horizon, $\|\mathbf{e}\|_{\mathbf{M}}^{2} \stackrel{\text { def }}{=} \mathbf{e}^{T} \mathbf{M e} . \boldsymbol{M}_{1}, \boldsymbol{M}_{2}, \boldsymbol{M}_{3}$ are weights that denote different control objectives, respectively. $J_{\min }$ is the cost function in quadratic form required to be minimized by the MPC solver, consisting of different control objectives. The first term in the equation represents the index of conversion efficiency, and rotor error $\mathbf{e}_{r}=\omega_{r}-\omega_{r}^{o p t}$. The second term denotes the output power fluctuations, $\Delta \mathbf{e}_{P_{e}}=\mathbf{P}_{e}(k)-\mathbf{P}_{e}(k-1)$. In the third term, the increment of control law at each sample time is substituted by the increment of compensation $K_{w}, \Delta \mathbf{K}_{w}=\mathbf{K}_{w}(k)-\mathbf{K}_{w}(k-1)$.

Because of the proposed MPPT framework, the control law at every sample time is calculated according to the special equation derived from Equation (9):

$$
\mathbf{u}(k)=\left(K_{o p t}^{\prime}-K_{w} \cdot \Gamma_{T w} / J\right) \cdot \delta \boldsymbol{\omega}_{r}(k)+N_{\text {gear }} \cdot K_{w} / J \cdot \delta \mathbf{T}_{e}(k)-K_{w} \cdot \Gamma_{T v} / J \cdot \delta \mathbf{V}_{w}(k)
$$

where $K_{\text {opt }}^{\prime}=0.5 \rho \pi R_{w}{ }^{5} C_{p \max } /\left(N_{\text {gear }} \cdot \lambda_{\text {opt }}^{3}\right)$.

In the proposed control, $K_{w}$ is constant within the entire control horizon. Therefore, the solving problem is simplified into computing the single parameter $K_{w}(k)$ instead of computing $\boldsymbol{u}(k), \ldots$, $\boldsymbol{u}\left(k+N_{c}-1\right)$, which is required in traditional MPC.

Ultra-short term prediction of wind speed is also required. Considering the emphasis is the MPPT method, wind forecasting is not studied in this paper. A wind prediction method can be found in [29], where the approximate precision of ultra-short term forecasting is up to more than $85 \%$. This means the uncertain dynamics of the predicted wind is small. Note that high precision of wind prediction is not necessary in this method, because just the information of variation trends of wind speed is just 
enough to consider the power fluctuations. The impacts of uncertain predictive wind on the control system are also discussed in Section 4.6.

\subsection{Proposed Dynamic Weights Regulation}

For a large-inertia wind turbine, the energy conversion efficiency can be kept at a high level under low wind speed fluctuations [3]. Only when the wind speed varies rapidly, the efficiency drops significantly, and this loss of conversion efficiency cannot be ignored. Therefore, different control objectives should be emphasized under different wind speed conditions. In this paper, a dynamic weights adjustment method is presented, rather than other preset weights methods. As shown in Figure 8, after uniformization, the three weights $M_{1}+M_{2}+M_{3}=1$. Weight $M_{3}$ is fixed which is designed to avoid oversize increments of the control law. Weights $M_{1}$ and $M_{2}$ are adjusted dynamically, and their sum is equal to $\left(1-M_{3}\right)$. If the second weight $M_{2}$ is determined, then $M_{1}=1-M_{2}-M_{3}$. The second weigh $M_{2}$ is expressed as:

$$
M_{2}=\frac{\bar{M}_{2} \cdot \hat{F}_{v}}{F_{v}}
$$

where $F_{v}=\sum_{j=1}^{N c}\left[V^{2}{ }_{w}(k+i)-V^{2}{ }_{w}(k+i-1)\right]^{2}$ denotes the statistical wind speed fluctuations within the control horizon. $\hat{F}_{v}$ is a preset threshold of wind fluctuations. $M_{2}$ varies around the preset parameter $\bar{M}_{2}$ depending on wind fluctuations. Ignoring wind variations, if $M_{2}$ is preset higher, the effect of power smoothing becomes more evident.

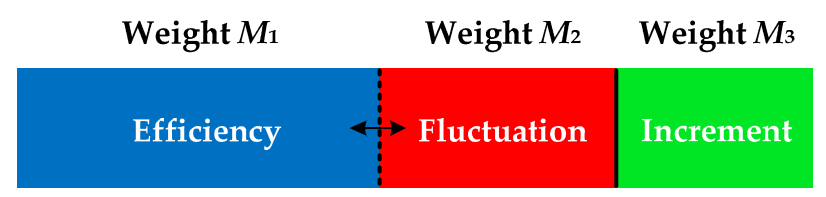

$M_{1}+M_{2}+M_{3}=1$

Figure 8. Schematic of dynamic weights in value function.

Since conversion efficiency can be kept at a high level when the wind changes slowly, $M_{2}$ is increased to be inclined to smoothing the power. Similarly, if the wind fluctuation gets higher, the conversion efficiency declines significantly, so $M_{1}$ has to be raised to enhance the efficiency.

\subsection{Stability Analysis}

Classical MPC is an open loop control system. The stability of the control system is guaranteed by terminal quality constraints. In this paper, the control law of $\boldsymbol{U}(k)$ is determined by both the proposed control framework and MPC solver. Therefore, the control system of the proposed method can be guaranteed so long as the control framework is stable.

The discrete system stability of control framework with different $K_{\mathrm{w}}$ is discussed. Ignoring wind speed turbulences, Equation (15) is rewritten as:

$$
\mathbf{u}(k)=\mathbf{G} \cdot \mathbf{X}(k)
$$

where $\mathbf{G}=\left[\left(K_{o p t}^{\prime}-K_{w} \cdot \Gamma_{T w} / J\right), \quad N_{\text {gear }} \cdot K_{w} / J\right]$.

Combining (13) and (17), the discrete state space equation is rewritten as:

$$
\left\{\begin{array}{c}
\mathbf{X}(k+1)=\mathbf{A}^{\prime} \mathbf{X}(k) \\
\mathbf{Y}(k)=\mathbf{C X}(k)
\end{array}\right.
$$

where $A^{\prime}=[A+B \cdot G]$. 
Figure 9 shows the locus of system eigenvalues with $K_{w}$ increasing from 0 to 70,000 . All the eigenvalues are located within the unit disk. According to the discrete system control theory [30], the control system is stable. With the increasing $K_{w}$, the eigenvalues move to the inner boundary of the unit disk. If the gain $K_{w}$ exceeds the limiting value, the eigenvalues would get out of the unit disk, and as a result, the stability of the control system cannot be ensured in this case and therefore, the stability of the proposed method can be guaranteed, so long as $K_{w}$ is limited within maximum value. This requirement can be satisfied by adding a limiter to ensure the reference torque to be non-negative.

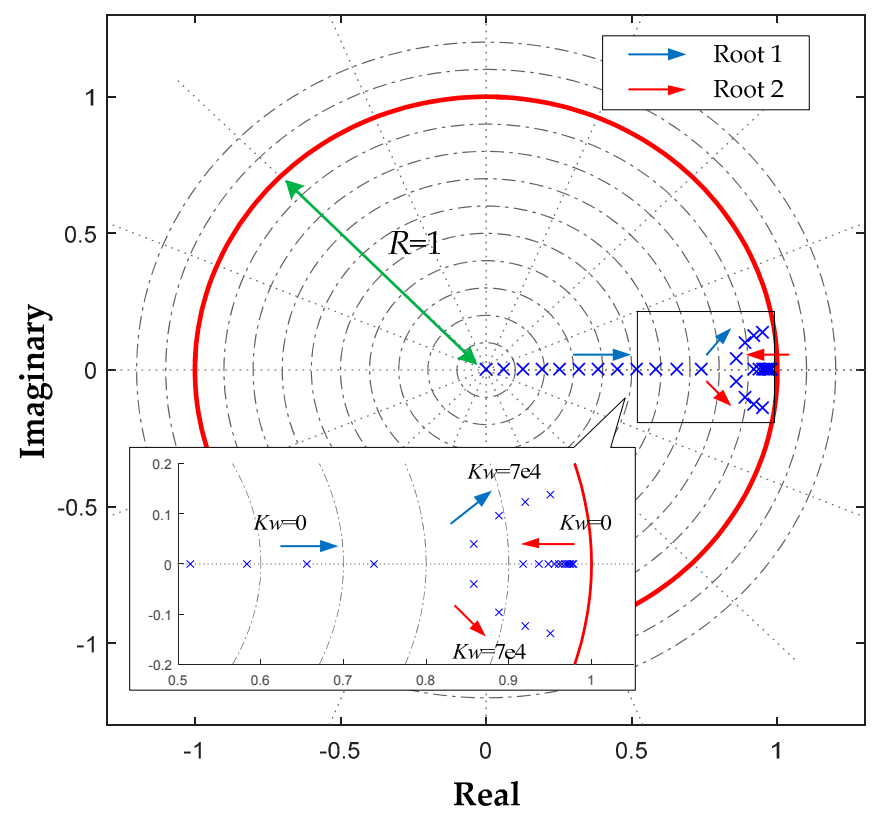

Figure 9. Eigenvalue locus of proposed control system.

\subsection{Robustness Analysis}

Uncertain parameters, including wind prediction uncertainties, and unmodeled dynamics, may bring about uncertain deviations of MPC output $K_{w}$ from the optimal value $\widetilde{K}_{w}$. Assume $\delta_{k}$ is the maximal uncertain deviation of MPC output, then the bounds of MPC output is expressed as:

$$
K_{w}^{ \pm}=\widetilde{K}_{w} \pm \delta_{k}
$$

Therefore, the MPC output $K_{w}$ at each sample time lies in the uncertain range $\left[K_{w}^{-}, K_{w}^{+}\right]$. As discussed in Section 4.5, the value $K_{w}$ determines the location of closed-loop eigenvalues. Eigenvalues are also called poles. The closed-loop eigenvalue location of within this uncertain range $\left[K_{w}^{-}, K_{w}^{+}\right]$is illustrated in Figure 10. All the eigenvalues are distributed in the colored region. The colored region depends on the uncertain range $\left[K_{w}^{-}, K_{w}^{+}\right]$. If the uncertain range is widened too much, then the colored region would overflow the stability boundary. As a result, eigenvalues may be located outside of the stability area, then the system becomes unstable. Therefore, to improve the robustness, uncertain range $\left[K_{w}^{-}, K_{w}^{+}\right]$of MPC output requires to be limited within stable area. In practice, MPC is capable of dealing with optimization problems with constraints. Thus uncertain range $\left[K_{w}^{-}, K_{w}^{+}\right]$ can be restricted by MPC solver, no matter how serious the uncertain parameters and unmodeled dynamics are. In general, the proposed control has good robust performance, and the stability is kept from uncertain parameters and unmodeled dynamics in MPC. 


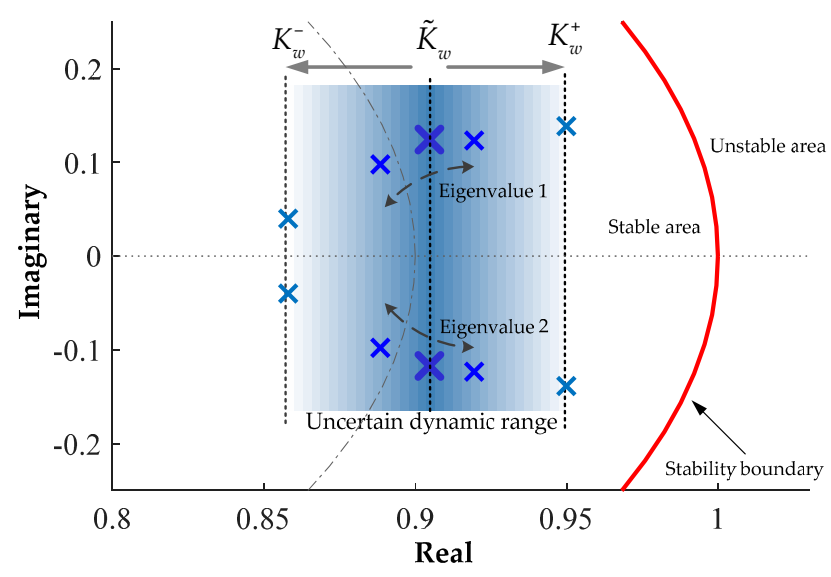

Figure 10. Closed-loop eigenvalue location within uncertain ranges of $K_{w}$.

\section{Simulation Studies}

To verify the effectiveness of the proposed method, comprehensive simulation studies are carried out based on the Wind Turbine Blockset Toolbox in Matlab/Simulink platform. The Blockset was developed by RISØ National Laboratory and Aalborg University, and it has been used as a general developer tool for other three simulation tools, namely: Saber, DIgSILENT and HAWC [31]. The Blockset has both mechanical and electrical model libraries that are accurate enough, especially the generator models, for this study. Considering the focus in this paper, the power grid that generator connects is assumed to be stable, and the power converters are regarded as average models.

Effective wind speed time series for simulations are generated by the wind model module in the Blockset that takes the tower shadow and the rotational turbulences into account. It is developed by RISØ National Laboratory based on the Kaimal spectra. The power spectra density for generated wind time series is calculated as shown in Figure 11. Wind direction is assumed to be fixed, thus yaw angle is set to zero. Wind shear is not taken into account, because the effective wind speed is calculated as an average value of the fixed-point wind speed over the whole rotor.

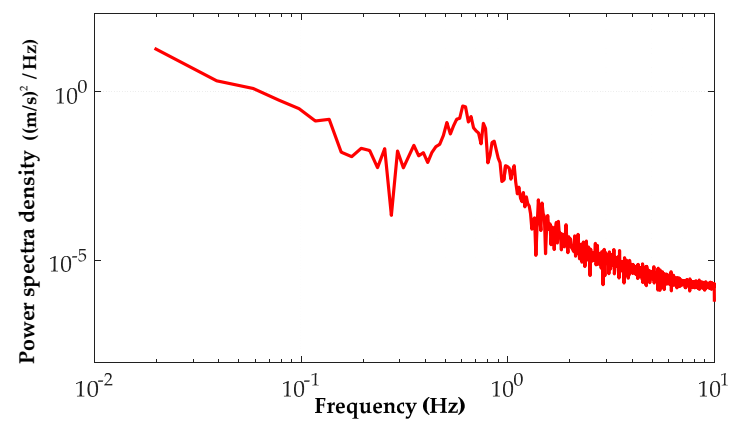

Figure 11. Power spectra density of wind speed.

The statistical approach of fluctuations is introduced in [1]. The main parameters in the simulations are listed as follows, $P_{\text {rated }}=2 \mathrm{MW}, R_{w}=40 \mathrm{~m}, V_{w_{-} \text {rated }}=11 \mathrm{~m} / \mathrm{s}, N_{\text {gear }}=83.531, \mathrm{~J}=56.27 \times 10^{5} \mathrm{~kg} \cdot \mathrm{m}^{2}$, $C_{p \max }=0.48, \lambda_{\text {opt }}=8.1[3,31]$.

\subsection{Case 1}

The first set of simulations is designed to investigate the control performance of the proposed method when the wind fluctuations change from high level to low level. The variations of wind fluctuations are achieved by changing the wind turbulence intensity [32]. 
As shown in Figure 12a, the wind speed is divided into two regions. At the beginning (Region I), the wind turbulence intensity is $20 \%$, and the fluctuation is large. In Region II, the wind turbulence intensity drops to $8 \%$. The proposed method is compared with a former method presented recently which has high conversion efficiency [5]. To compare them with each other impartially, the average conversion efficiency of two methods are tuned to be the same in Region I, as shown in Table 1. Parameters in (16) are, $\bar{M}_{2}=0.15, \hat{F}_{v}=0.0045, M_{3}=0.3$. This simulation reveals two main aspects of advantages. Firstly, in Region I, the proposed method has lower power fluctuations when the average efficiencies of the two methods are almost the same. Secondly, in Region II, when wind speed varies gently, the output power of the proposed method is much smoother, which is nearly one fifth of former method, as shown in Table 1 and Figure 12b. However, the conversion efficiency is also kept at a high level. Although the efficiency of the proposed method is 0.0002 smaller than former method as shown in Table 1, this difference is so tiny that can be ignored. It is noted that the power fluctuation of the proposed method is higher than that of the former method near time $=95 \mathrm{~s}$ in Figure 12b. The reason is that the wind speed drops significantly at this time. As a result, to avoid conversion efficiency dropping too much, control objective tends to restrain efficiency from increasing, as shown in Figure 12d. This also indicates the proposed method has the capability to adjust to different wind situations dynamically considering both conversion efficiency and power smoothing.
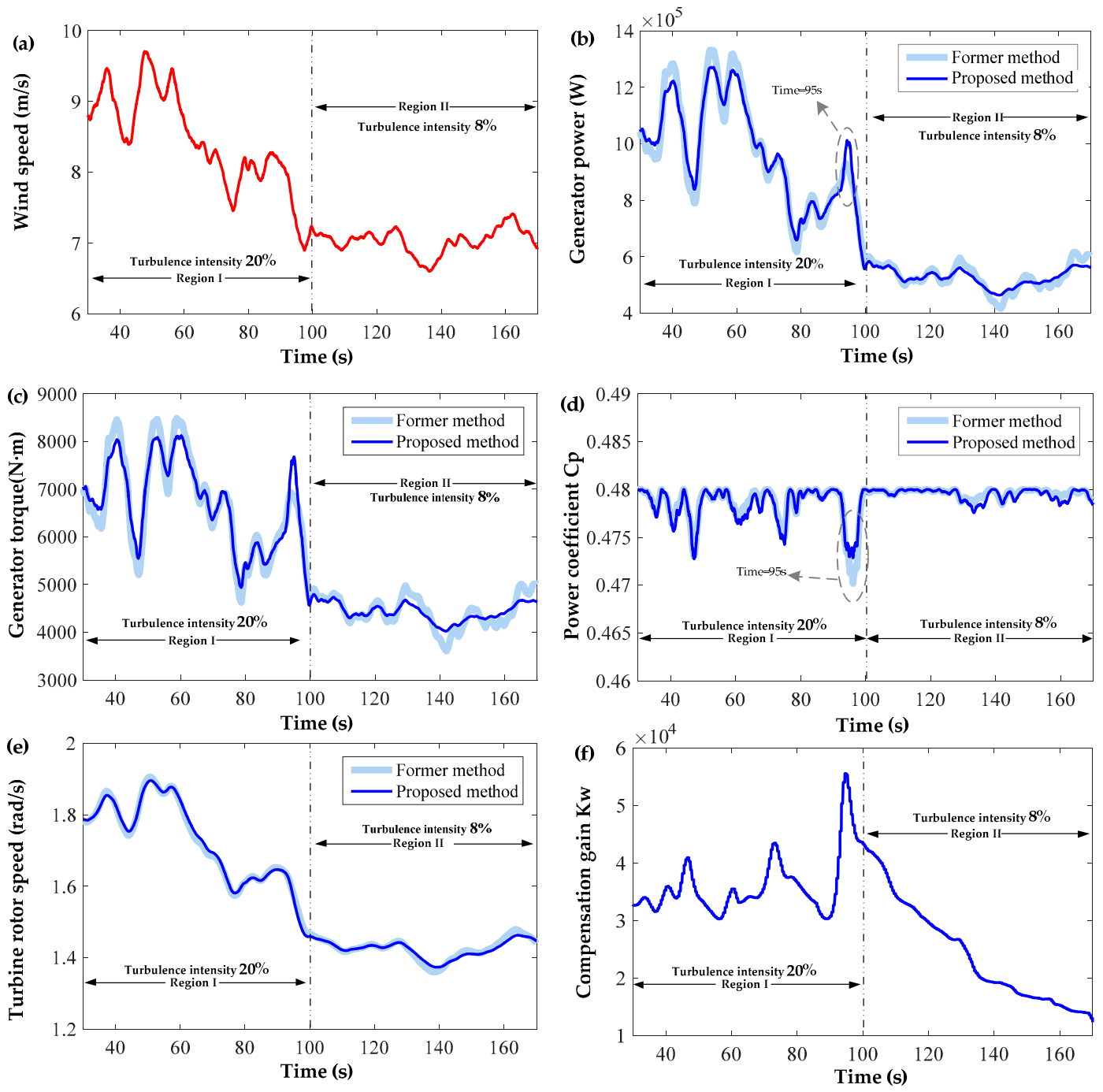

Figure 12. Simulation results under low and high wind fluctuations, (a) wind speed; (b) generator power; (c) generator torque; (d) conversion efficiency; (e) rotor speed; (f) compensation gain. 
Table 1. Statistics of efficiency and fluctuations.

\begin{tabular}{ccccc}
\hline \multirow{2}{*}{ Methods } & \multicolumn{2}{c}{ Region I } & \multicolumn{2}{c}{ Region II } \\
\cline { 2 - 5 } & Average $C_{p}$ & Fluctuations & Average $C_{p}$ & Fluctuations \\
\hline Former & 0.4783 & $1.884 \times 10^{11}$ & 0.4796 & $1.140 \times 10^{10}$ \\
Proposed & 0.4783 & $1.279 \times 10^{11}$ & 0.4794 & $2.541 \times 10^{9}$ \\
\hline
\end{tabular}

\subsection{Case 2}

Instead of separating the wind fluctuations into two levels as in Case 1, different wind fluctuation conditions are all taken into account to verify the proposed method in depth. Since different parameters in control systems lead to different control performances, it is essential to impartially compare the control performance of those methods under the same operation conditions. Therefore, as shown in Table 2, to compare the capability of power smoothing, controller parameters are tuned to guarantee that two methods have almost the same conversion efficiency. Parameters in (16) are as follows, $\bar{M}_{2}=0.15, \hat{F}_{v}=0.0045, M_{3}=0.3$.

Table 2. Statistics of efficiency and fluctuations.

\begin{tabular}{ccc}
\hline Method & Average $C_{p}$ & Fluctuations \\
\hline Former & 0.4785 & $5.806 \times 10^{11}$ \\
Proposed & 0.4785 & $5.041 \times 10^{11}$ \\
\hline
\end{tabular}

The simulation results illustrated in Figure 13 show that the generator power of the proposed method is smoother than that of the former method. The power fluctuation of the proposed method is reduced by $13.18 \%$ compared with the former method within the entire simulation time.
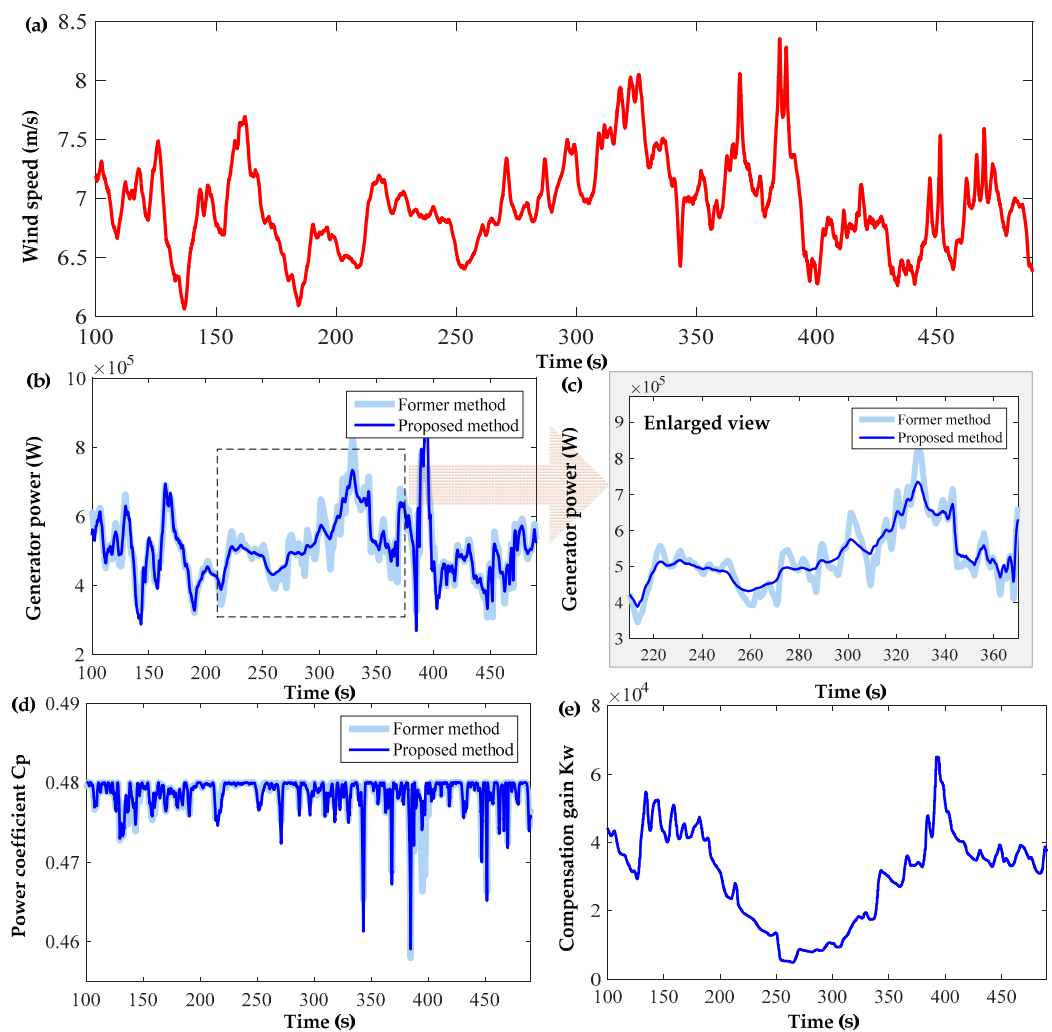

Figure 13. Simulation results under different wind fluctuations, (a) wind speed; (b) generator power; (c) enlarged view of generator power; (d) conversion efficiency; (e) compensation gain. 
However, the conversion efficiency of the proposed method is not reduced. Figure 13c is the enlarged view of generator power in the dashed region of Figure 13b. In general, this set of simulations reveals that the proposed method has better power smoothing capability than that of other efficiency enhancement methods. In addition, the parameters of the control system require no changes to adjust to the variations of wind speed.

\subsection{Case 3}

The third set of simulations is designed to prove the advantages of the proposed dynamic weights in Equation (14). The two compared methods have the same control framework proposed in this paper. The only difference between them is that the weights of the proposed method are changed dynamically, and the weights of the compared one are constant. We respectively name the two methods after proposed method with dynamical weights and proposed method with constant weights in the following analysis. The settings of weights are listed in Table 3.

Table 3. Parameters in value function.

\begin{tabular}{cc}
\hline Proposed Method & Parameters \\
\hline Dynamic weights & $\hat{F}_{v}=0.004, \bar{M}_{2}=0.2, M_{3}=0.3$ \\
Constant weights & $M_{1}=0.5, M_{2}=0.2, M_{3}=0.3$ \\
\hline
\end{tabular}

As shown in Figure 14a, the wind fluctuation changes gradually from low level to high level along with the time. According to [3], the conversion efficiency loss of a large-inertia wind turbine under low wind fluctuation condition is small, but the loss increases significantly when the wind speed varies rapidly. Thus, when the wind speed varies slowly, it is better to reduce $K_{w}$ to utilize the large inertia to smooth output power, and avoid extra fluctuations.
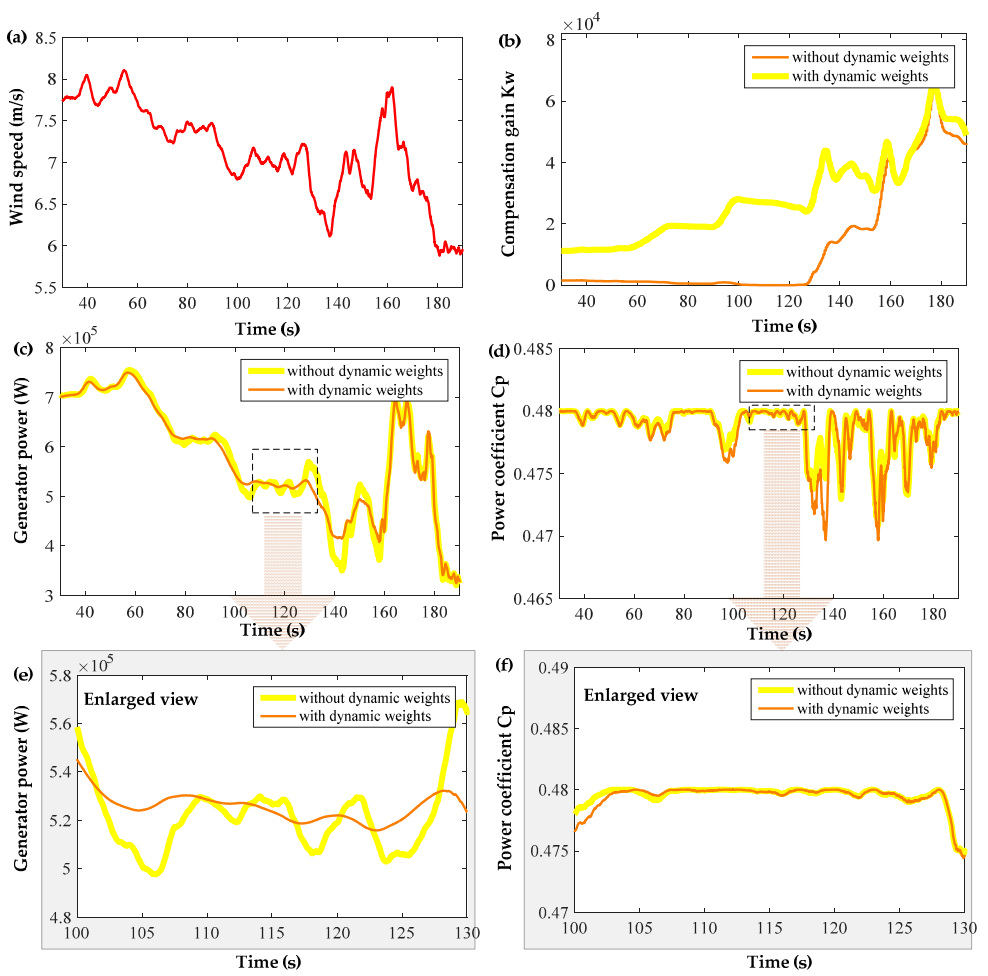

Figure 14. Comparison results with different weights preset methods, (a) wind speed; (b) compensation gain; (c) generator power; (d) conversion efficiency; (e) enlarged view of generator power; (f) enlarged view of conversion efficiency. 
As illustrated in Figure 14b, in the low wind fluctuation region (30-130 s), the $K_{w}$ of the proposed method with dynamic weights is smaller than that of the method with constant weights. Therefore, the proposed method with dynamic weights obtains better power smoothing performance with almost no efficiency losses. This advantage becomes more evident when the wind fluctuation becomes lower, such as in the very low fluctuation region (100-130 s), where the generator power of the method with dynamic weights is much smoother, but the efficiencies of the two methods are almost the same, as shown in Figure 14e,f.

In the high wind fluctuation region (160-190 s), the conversion efficiency would decline significantly. Thus, it is essential to increase $M_{1}$ and reduce $M_{2}$ to enhance the efficiency. As a result, $K_{w}$ of the proposed method with dynamic weights is raised, which is close to the proposed method without dynamic weights.

The advantages above benefit from the dynamic weights within the entire simulation time. Different from the fixed weights $\left(M_{1}=0.5, M_{2}=0.2\right)$ in the proposed method with constant weights, the weights in the proposed method change along with the time to get better control performance. The variations of weights in the proposed method with dynamic weights are illustrated in Figure 15. The vertical axis denotes the relative proportion of each weight at each sample time. Here, $M_{1}+M_{2}+M_{3}=1, M_{3}=0.3$. For instance, at time $170 \mathrm{~s}$, the weight $M_{1}$ is $0.33, M_{2}$ is $0.37, M_{3}$ is 0.3 . Because the weight $M_{3}$ is fixed, the sum of $M_{1}$ and $M_{2}$ is 0.7 .

Note that dynamic weights may cause a few limited efficiency losses in some special situations. For example, as shown in Figure $14 \mathrm{~d}$, due to the step size limitation of $K_{w}$, the efficiency of the proposed method with dynamic weights is a little lower than that of the compared method around time $=135 \mathrm{~s}$ when the wind fluctuation changes suddenly, but this also means a lower power fluctuation. This special situation can also be alleviated by decreasing the weight $M_{3}$ in Equation (14).

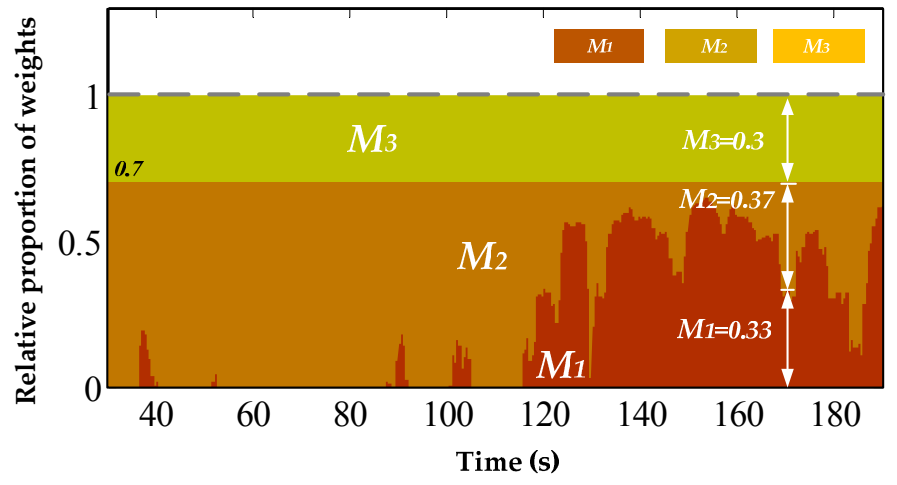

Figure 15. Weights variations of the proposed method with dynamic weights.

In general, the two compared methods based on proposed MPC approach both have high efficiency and low fluctuations, but the power smoothing performance of the proposed method with dynamic weights is better than that of the method with constant weights at almost no cost of conversion efficiency.

\subsection{Computing Time}

The execution time of MPC solver within one sample period is calculated to verify the capability of real-time implementation. The environment information for the execution program is shown in Table 4. MATLAB is set to run the program in single thread mode. As shown in Table 5, MPC solver takes $0.1737 \mathrm{~s}$ at each sample time to execute the optimization problem, which is much shorter than the sample period $1 \mathrm{~s}$. This indicates that the proposed MPC is fully capable of real-time implementation. Furthermore, the fast development of embedded processors, such as digital signal processing (DSP) 
and field-programmable gate array (FPGA), supplies a practicable computing platform for real-time execution [33].

Table 4. Computing environment.

\begin{tabular}{cc}
\hline CPU & Intel Core i3-2370M (2.4 GHz) (Single Core Mode) \\
RAM & $4 \mathrm{~GB}$ \\
Operating system & Window 7 (32 bit) \\
Platform & MATLAB R2012a \\
\hline
\end{tabular}

Table 5. Computing time of MPC solver.

\begin{tabular}{cc}
\hline Parameter & Value \\
\hline Sample period $T$ & $1(\mathrm{~s})$ \\
Control horizon $N_{c}$ & 5 \\
Solver & Sequence Quadratic Program \\
Computing time & $0.1737(\mathrm{~s})$ \\
\hline
\end{tabular}

\section{Conclusions}

This paper presents a flexible MPPT control method to balance captured energy efficiency and generator power smoothing for large-inertia WECSs. A new control framework is proposed by adaptively compensating the torque reference value. The compensation was determined by the proposed model predictive control approach with dynamic weights in the cost function, which improved control performance. The computational burden of the MPC solver was reduced by transforming the cost function representation. The main contributions of this paper are summarized as follows:

- Analysis reveals that a larger wind turbine inertia brings difficulties to balance energy conversion efficiency and generator power smoothing. To achieve the same conversion efficiency, a larger inertia causes higher power fluctuations.

- The proposed method not only keeps the conversion efficiency at a high level, but also reduces power fluctuations as much as possible. Emphasis of control objectives is regulated dynamically. Conversion efficiency is attached importance when it is about to decline too much. Power smoothing is taken more into account if the wind varies slowly, and efficiency is kept at a high level.

- Unlike the classical MPC used for wind turbine control, MPC in this paper is only employed to determine the compensating gain. The system stability is totally based on the proposed control framework, rather than the stability of MPC itself. Theoretical analysis proves that the control system is stable, so long as the compensating gain in proposed control framework is kept within the maximal value.

- The robustness of the system is guaranteed by the proposed control, the stability avoids uncertain parameters and unmodeled dynamics in the MPC.

- Simulation studies are carried out to prove that the proposed method has good control performance. The computing time of the MPC solver at each sampling time is short enough to satisfy the requirements of real-time implementation in engineering.

In general, these good capabilities of the proposed method, such as high conversion efficiency and low power fluctuations can increase the wind farm profits and improve power system reliability.

Acknowledgments: This work was supported in part by the National Basic Research Program of China (973 Program) (Grant No. 2012CB215203), National Nature Science Foundation of China (Grant No. 51606033) and Beijing Postgraduate Joint-supervision Cooperate Project. 
Author Contributions: Hongmin Meng and Tingting Yang conceived the control strategy, designed and performed simulations. Ji-zhen Liu provided key advices of proposed strategy. Zhongwei Lin analyzed the data. Hongmin Meng wrote and revised the paper.

Conflicts of Interest: The authors declare no conflict of interest.

\section{Abbreviations}

$\begin{array}{ll}\text { MPPT } & \text { Maximum power point tracking } \\ \text { MOO } & \text { Multi-objective optimization } \\ \text { FIS } & \text { Fuzzy inference system } \\ \text { WECS } & \text { Wind energy conversion system } \\ \text { MPC } & \text { Model predictive control } \\ \text { OTC } & \text { Optimal torque control } \\ \text { GSC } & \text { Generator side converter } \\ \text { PI } & \text { Proportional-Integral } \\ \text { OP } & \text { Operation point } \\ \text { MIMO } & \text { Multi-input multi-output } \\ \text { QP } & \text { Quadratic programming }\end{array}$

\section{References}

1. Simani, S. Overview of modelling and advanced control strategies for wind turbine systems. Energies 2015, 8, 12374. [CrossRef]

2. Phan, D.-C.; Yamamoto, S. Maximum energy output of a dfig wind turbine using an improved mppt-curve method. Energies 2015, 8, 11718-11736. [CrossRef]

3. Liu, J.; Meng, H.; Hu, Y.; Lin, Z.; Wang, W. A novel mppt method for enhancing energy conversion efficiency taking power smoothing into account. Energy Conv. Manag. 2015, 101, 738-748. [CrossRef]

4. Hussain, J.; Mishra, M.K. Adaptive maximum power point tracking control algorithm for wind energy conversion systems. IEEE Trans. Energy Conv. 2016, 31, 697-705. [CrossRef]

5. Kim, K.H.; Van, T.L.; Lee, D.C.; Song, S.H.; Kim, E.H. Maximum output power tracking control in variable-speed wind turbine systems considering rotor inertial power. IEEE Trans. Ind. Electron. 2013, 60, 3207-3217. [CrossRef]

6. Uehara, A.; Pratap, A.; Goya, T.; Senjyu, T.; Yona, A.; Urasaki, N.; Funabashi, T. A coordinated control method to smooth wind power fluctuations of a pmsg-based wecs. IEEE Trans. Energy Conv. 2011, 26, 550-558. [CrossRef]

7. Wei, C.; Zhang, Z.; Qiao, W.; Qu, L. An adaptive network-based reinforcement learning method for MPPT control of pmsg wind energy conversion systems. IEEE Trans. Power Electron. 2016, 31, 7837-7848. [CrossRef]

8. Iyasere, E.; Salah, M.H.; Dawson, D.M.; Wagner, J.R.; Tatlicioglu, E. Robust nonlinear control strategy to maximize energy capture in a variable speed wind turbine with an internal induction generator. J. Control Theory Appl. 2012, 10, 184-194. [CrossRef]

9. Boukhezzar, B.; Siguerdidjane, H. Nonlinear control of a variable-speed wind turbine using a two-mass model. IEEE Trans. Energy Conv. 2011, 26, 149-162. [CrossRef]

10. Dawei, Z.; Lie, X.; Williams, B.W. Model-based predictive direct power control of doubly fed induction generators. IEEE Trans. Power Electron. 2010, 25, 341-351. [CrossRef]

11. Sguarezi Filho, A.J.; de Oliveira Filho, M.E.; Filho, E.R. A predictive power control for wind energy. IEEE Trans. Sustain. Energy 2011, 2, 97-105.

12. Calle-Prado, A.; Alepuz, S.; Bordonau, J.; Nicolas-Apruzzese, J.; Cortes, P.; Rodriguez, J. Model predictive current control of grid-connected neutral-point-clamped converters to meet low-voltage ride-through requirements. IEEE Trans. Ind. Electron. 2015, 62, 1503-1514. [CrossRef]

13. Yaramasu, V.; Wu, B. Predictive control of a three-level boost converter and an npc inverter for high-power pmsg-based medium voltage wind energy conversion systems. IEEE Trans. Power Electron. 2014, 29, 5308-5322. [CrossRef] 
14. Kou, P.; Liang, D.; Gao, F.; Gao, L. Coordinated predictive control of dfig-based wind-battery hybrid systems: Using non-gaussian wind power predictive distributions. IEEE Trans. Energy Conv. 2015, 30, 681-695. [CrossRef]

15. Evans, M.A.; Cannon, M.; Kouvaritakis, B. Robust MPC tower damping for variable speed wind turbines. IEEE Trans. Control Syst. Technol. 2015, 23, 290-296. [CrossRef]

16. Spencer, M.D.; Stol, K.A.; Unsworth, C.P.; Cater, J.E.; Norris, S.E. Model predictive control of a wind turbine using short-term wind field predictions. Wind Energy 2013, 16, 417-434. [CrossRef]

17. Barlas, T.; van der Veen, G.; van Kuik, G. Model predictive control for wind turbines with distributed active flaps: Incorporating inflow signals and actuator constraints. Wind Energy 2012, 15, 757-771. [CrossRef]

18. Soliman, M.; Malik, O.P.; Westwick, D.T. Multiple model predictive control for wind turbines with doubly fed induction generators. IEEE Trans. Sustain. Energy 2011, 2, 215-225. [CrossRef]

19. Soliman, M.; Malik, O.P.; Westwick, D.T. Multiple model mimo predictive control for variable speed variable pitch wind turbines. In Proceedings of the American Control Conference (ACC), Baltimore, MD, USA, 30 June-2 July 2010; pp. 2778-2784.

20. Mayne, D.Q.; Rawlings, J.B.; Rao, C.V.; Scokaert, P.O. Constrained model predictive control: Stability and optimality. Automatica 2000, 36, 789-814. [CrossRef]

21. Bououden, S.; Chadli, M.; Filali, S.; El Hajjaji, A. Fuzzy model based multivariable predictive control of a variable speed wind turbine: Lmi approach. Renew. Energy 2012, 37, 434-439. [CrossRef]

22. Jain, A.; Schildbach, G.; Fagiano, L.; Morari, M. On the design and tuning of linear model predictive control for wind turbines. Renew. Energy 2015, 80, 664-673. [CrossRef]

23. Kusiak, A.; Li, W.; Song, Z. Dynamic control of wind turbines. Renew. Energy 2010, 35, 456-463. [CrossRef]

24. Can, H.; Fangxing, L.; Zhiqiang, J. Maximum power point tracking strategy for large-scale wind generation systems considering wind turbine dynamics. IEEE Trans. Ind. Electron. 2015, 62, 2530-2539.

25. Bianchi, F.D.; Battista, H.D.; Mantz, R.J. Wind Turbine Control Systems. Principles, Modelling and Gain Scheduling Design; Springer: London, UK, 2007.

26. Abedini, A.; Mandic, G.; Nasiri, A. Wind power smoothing using rotor inertia aimed at reducing grid susceptibility. Int. J. Power Electron. 2008, 1, 227-247. [CrossRef]

27. Varzaneh, S.G.; Gharehpetian, G.B.; Abedi, M. Output power smoothing of variable speed wind farms using rotor-inertia. Electr. Power Syst. Res. 2014, 116, 208-217. [CrossRef]

28. Nasiri, M.; Milimonfared, J.; Fathi, S. Modeling, analysis and comparison of tsr and otc methods for mppt and power smoothing in permanent magnet synchronous generator-based wind turbines. Energy Conv. Manag. 2014, 86, 892-900. [CrossRef]

29. Pourmousavi Kani, S.A.; Ardehali, M.M. Very short-term wind speed prediction: A new artificial neural network-markov chain model. Energy Conv. Manag. 2011, 52, 738-745. [CrossRef]

30. Zheng, A.; Morari, M. Stability of model predictive control with mixed constraints. IEEE Trans. Autom. Control 1995, 40, 1818-1823. [CrossRef]

31. Iov, F.; Hansen, A.D.; Blaabjerg, F. Wind Turbine Blockset in Matlab/Simulink; Institute of Energy Technology, Aalborg University: Aalborg, Denmark, 2004.

32. Hansen, K.S.; Barthelmie, R.J.; Jensen, L.E.; Sommer, A. The impact of turbulence intensity and atmospheric stability on power deficits due to wind turbine wakes at Horns Rev wind farm. Wind Energy 2012, 15, 183-196. [CrossRef]

33. Gulbudak, O.; Santi, E. Fpga-based model predictive controller for direct matrix converter. IEEE Trans. Ind. Electron. 2016, 63, 4560-4570. [CrossRef]

(C) 2017 by the authors. Licensee MDPI, Basel, Switzerland. This article is an open access article distributed under the terms and conditions of the Creative Commons Attribution (CC BY) license (http:/ / creativecommons.org/licenses/by/4.0/). 